\title{
Mielografia lombar no diagnóstico de extrusão do disco intervertebral toracolombar em cães: estudo prospectivo
}

\section{Lumbar myelography in the diagnosis of thoracolumbar intervertebral disk extrusion in dogs: prospective study}

\author{
Paulo Vinicius Tertuliano Marinho ${ }^{1 *}$; Bruno Watanabe Minto ${ }^{2}$; Carolina Camargo \\ Zani $^{3}$; Érica Siqueira de Souza ${ }^{4}$; Nathália Helena Pereira da Silva Dal Pietro5; \\ João Augusto Leonel de Souza ${ }^{6}$; Júlio Carlos Canola ${ }^{7}$; Andrigo Barboza De Nardi ${ }^{7}$
}

\section{Resumo}

\begin{abstract}
A extrusão do disco toracolombar é a causa mais comum de compressão extradural da medula espinhal em cães. A mielografia é uma das técnicas mais comumente realizadas para o diagnóstico desta afecção. Objetivou-se avaliar a aplicabilidade e efetividade da técnica de mielografia lombar no diagnóstico de extrusão do disco intervertebral toracolombar em cães, bem como suas principais complicações. Foram utilizados 20 animais que ao exame neurológico apresentaram localização da lesão na região toracolombar e que tiveram indicação de mielografia como método diagnóstico complementar e confirmação do diagnóstico de extrusão do disco no trans-operatório. Avaliou-se a precisão do exame na localização e lateralização da lesão, e as complicações associadas ao procedimento. A mielografia lombar teve acurácia de $95 \%$ e $60 \%$ para a localização e a lateralização da lesão respectivamente, com complicações mínimas.
\end{abstract}

Palavras-chave: Diagnóstico, medula espinhal, punção lombar

\begin{abstract}
Thoracolumbar disk extrusion is the most common cause of extradural compression of the spinal cord in dogs. Myelography is one of the most commonly performed techniques for the diagnosis of this affection. This study aimed to evaluate the applicability and effectiveness of lumbar myelography in the diagnosis of thoracolumbar intervertebral disk extrusion in dogs, as well as its major complications. Twenty dogs were used in this study. Animals were included when neurological examination suggested thoracolumbar spine lesion, myelography was used as a complementary diagnostic method, and diagnosis of disk extrusion was surgically confirmed. The accuracy of the exam to predict location and lateralization of extruded disk material were evaluated, as well as complications associated to the procedure. Lumbar myelography exhibited $95 \%$ and $60 \%$ accuracy for location and lateralization of the lesion, respectively, with minimal complications.
\end{abstract}

Key words: Diagnosis, spinal cord, lumbar puncture

1 Discente de Mestrado, Pesquisador, Universidade Estadual de Londrina, UEL, Londrina, PR. E-mail: paulo.veter@gmail.com

2 Prof., Faculdade de Ciências Agrárias e Veterinária, FCAV/UNESP, Jaboticabal, SP. E-mail: brunobtu@yahoo.com.br

3 Discente de Mestrado, Pesquisador, UEL, Londrina, PR. E-mail: carolzani_vet@yahoo.com.br

${ }^{4}$ Médica Veterinária, Residente do Dept ${ }^{\mathfrak{D}}$ de Cirurgia Veterinária, Universidade Estadual Paulista, FCAV/UNESP, Jaboticabal, SP. E-mail: souza.ericacv@yahoo.com.br

5 Médica Veterinária Autônoma. E-mail: nathaliadalpietro@hotmail.com

${ }^{6}$ Médico Veterinário, Residente do Dept ${ }^{\circ}$ de Cirurgia Veterinária, UNESP, Botucatu, SP. E-mail: desouza.ja@gmail.com

7 Profs., Faculdade de Ciências Agrárias e Veterinária, FCAV/UNESP, Jaboticabal, SP. E-mail: canola@fcav.unesp.br; andrigobarboza@yahoo.com.br

Autor para correspondência 


\section{Introdução}

A mielografia é uma técnica de neuroimagem comumente utilizada no diagnóstico de distúrbios compressivos envolvendo a medula espinhal em cães e gatos (DA COSTA; PARENT; DOBSON, 2011). A extrusão do disco toracolombar é a causa mais comum de compressão extradural da medula espinhal em cães de pequeno porte (JESEN, 2001; LEVINE, 2008). Como a maioria das extrusões discais são lateral ou ventrolateral em relação a medula espinhal, a localização cirucunferencial é essencial para orientar em relação a técnica e o lado da descompressão cirúrgica (TANAKA; NAKAYAMA; TAKASE, 2004; BESALTI et al., 2006; NAUDE; LAMBRECHTS; WAGNER 2008).

A mielografia possibilita a avaliação do espaço subaracnóide espinhal e das margens externas da medula espinhal (ROBERTS; SELCER, 1993), sendo utilizada para demonstrar lesões internas ou extrínsecas à medula espinhal, mas que causam compressão sobre a mesma (KEALY; McALLISTER, 2005).

A precisão relatada com uso da mielografia é de 72 a 97\% para a localização da lesão e 53 a 100\% para a lateralização da lesão nas extrusões discais (KIRBERGER; ROOS; LUBBE, 1992; SCHULZ et al., 1998; GIBBONS et al., 2006; BOS et al., 2007; NEWCOMB et al., 2012).

Existem duas formas mais comuns para realização de punção e injeção de contraste para realização da mielografia, a cisterna magna e o espaço intervertertebral lombar entre as vértebras lombares 5 e 6 (L5-L6) (PUGGIONI et al., 2006). A mielografia através da punção na cisterna magna é condiderada um procedimento de fácil realização e eficaz na localização da lesão, porém a acurácia na identificação das lesões toracolombares é melhor quando a punção lombar é realizada (SHARP; WHEELER, 2005; BRISSON, 2010). A principal complicação associada à punção cervical é a penetração da agulha espinhal na medula cervical ou no tronco encefálico levando a parada respiratória, além do aumento considerável do risco de convulsões pós-exame (DA COSTA; PARENT; DOBSON, 2011). A mielografia lombar, por sua vez, é tecnicamente mais exigente do que mielografia cervical, porém é mais acurada na identificação de lesões toracolombares, e apresenta menor risco de convulsões (OLBY; HOULTON, 1994). De acordo com McCartney (1997), punções até T13-L1 podem ser realizadas e produzir uma mielografia diagnóstica, no entanto, punções craniais a L5 podem levar a canalograma em 4,4 a $20 \%$ dos casos, com piora potencial dos déficitis neurológicos (KIRBERGER; ROOS; LUBBE, 1992; McCARTNEY, 1997). Projeções radiográficas ventrodorsais, laterais e oblíquas da área requerida devem ser obtidas imediatamente após a injeção de contraste (BRISSON, 2010). Apesar da mielografia não ser indicada em pacientes com pressão intracraniana aumentada, se esta for necessária, pode ser realizada na região lombar, pois diminuem os riscos de herniação cerebelar (SHARP; WHEELER, 2005). A principal complicação associada a técnica lombar é a injeção do contraste no espaço epidural gerando imagem de epidurografia, que não delimita o local da lesão com exatidão (SHARP; WHEELER, 2005).

Para minimizar os efeitos neurotóxicos associados à mielografia, o agente de contraste ideal deve ser farmacologicamente inerte, miscível com líquido encéfaloraquidiano, solúvel em água, e radiopaco numa concentração isotônica (DA COSTA; PARENT; DOBSON, 2011). O meio de contraste mais utilizado na medicina veterinária é o iohexol (DA COSTA; PARENT; DOBSON, 2011). A incidência de convulsões pós-mielografias com iohexol varia de 0 a $10 \%$ e tem sido associada com o peso do paciente (pacientes maiores), o volume de injeção de contraste (volumes maiores), a injeção na cisterna magna, localização da lesão (cervical mais provável), sexo (maior risco em machos) e raça (maior risco em dobermans pinchers) (WHEELER; DAVIES, 1985; LEWIS; HOSGOOD, 1992; OLBY; HOULTON, 1994; ALLAN; WOOD, 1988; 
BARONE et al., 2002; DA COSTA; PARENT; DOBSON, 2011; NEWCOMB et al., 2012). A injeção do contraste em L5 e L6 para a mielografia em cães mais leves que $20 \mathrm{~kg}$ está associada com taxas mais baixas de convulsão pós-mielografia (BARONE et al., 2002; ISRAEL et al., 2009).

Na punção lombar, os riscos de efeitos adversos e lesões iatrogênicas são menores do que a punção na cisterna magna (KISHIMOTO et al., 2004). Esta afirmação estimulou o desenvolvimento da presente pesquisa, de forma a realizar-se a mielografia sem prejuízos ao seu valor diagnóstico e minimizandose as complicações inerentes ao procedimento.

Objetivou-se estudar a aplicação e efetividade da técnica de mielografia lombar no diagnóstico de extrusão do disco intervertebral toracolombar em cães, bem como suas possíveis complicações.

\section{Material e Métodos}

Foram utilizados 20 cães, de raças e idades variadas, que ao exame neurológico apresentaram localização da lesão neurológica na região toracolombar e que tiveram indicação de mielografia como método de diagnóstico complementar. Todos os pacientes foram provenientes do atendimento clínico hospitalar de rotina do serviço de clínica cirúrgica de pequenos animais. Todos os pacientes foram selecionados previamente por meio dos exames clínico e neurológico completos.

Os pacientes foram submetidos à anestesia geral intravenosa (propofol na dose de 3 a $5 \mathrm{mg}$ / $\mathrm{kg}$ associado a $0,7 \mathrm{mg} / \mathrm{kg}$ de diazepam). Após a intubação endotraqueal, os pacientes foram mantidos em oxigênio a 100\%. Bolus de propofol foram reaplicados para manutenção da anestesia segundo a necessidade.

Foi realizada tricotomia ampla da região lombar, desde a região da primeira vértebra lombar até a última vértebra sacral seguida de antissepsia cirúrgica.
Primeiramente, foi realizado o exame radiográfico simples da coluna vertebral a ser avaliada, nas projeções ventrodorsal e laterolateral. Ato contínuo, os pacientes foram submetidos à punção lombar por meio da inserção de uma agulha espinhal entre os espaços intervertebrais L5-L6, estando adequadamente posicionados em decúbito lateral com flexão moderada do tronco em sentido cranial. A agulha espinhal foi introduzida ligeiramente caudolateralmente ao processo espinhoso da $5^{\mathrm{a}}$ vértebra lombar e direcionada cranioventralmente em um ângulo de $45^{\circ}$. Em seguida, a agulha foi transpassada através do ligamento amarelo e a dura-máter e concomitantemente observou-se movimentação da cauda do paciente e a saída do líquido cerebroespinhal (LCE), sendo o meio de contraste injetado. Caso não tenha sido observada a presença do LCE a agulha foi avançada até o assoalho ventral do canal vertebral e procedeuse com discreto retorno da mesma para o espaço subaracnóide (1 a 1,5 mm).

O meio de contraste utilizado foi o iohexol, na dose de $0,35 \mathrm{ml} / \mathrm{kg}$. Imediatamente após a injeção lenta do contraste (em aproximadamente 3 minutos), foram realizadas radiografias nas projeções lateral (Figura 1), ventrodorsal e oblíquas (Figura 2) para evidenciar a localização da afecção medular.

Realizou-se a avaliação da mielografia por observação das colunas de contraste que delimitaram a medula espinhal. Normalmente, essas colunas apresentam-se como finas linhas paralelas e homogêneas.

Os parâmetros avaliados nas imagens foram: localização da lesão, lateralização da lesão e complicações. A localização da lesão foi realizada por visualização da atenuação ou desvio da coluna de contraste na projeção lateral e ventrodorsal sugerindo compressão extradural como diagnóstico de doença do disco intervertebral. Já, as projeções ventrodorsais e oblíquias sugeriram a lateralização da lesão. 
Possíveis complicações como o derrame histórico, evolução da doença e pela característica subdural, canalograma e epidurografia também do material encontrado dentro do canal vertebral no foram registrados. momento da cirurgia descompressiva.

Em todos os pacientes, o diagnóstico de extrusão do disco intervertebral foi confirmado com base no

Figura 1. Imagem de mielografia lombar com elevação da coluna de contraste (seta) entre a décima primeira (T11) e décima segunda (T12) vértebras torácicas, que evidencia a localização da lesão em um cão com extrusão do disco intervertebral.

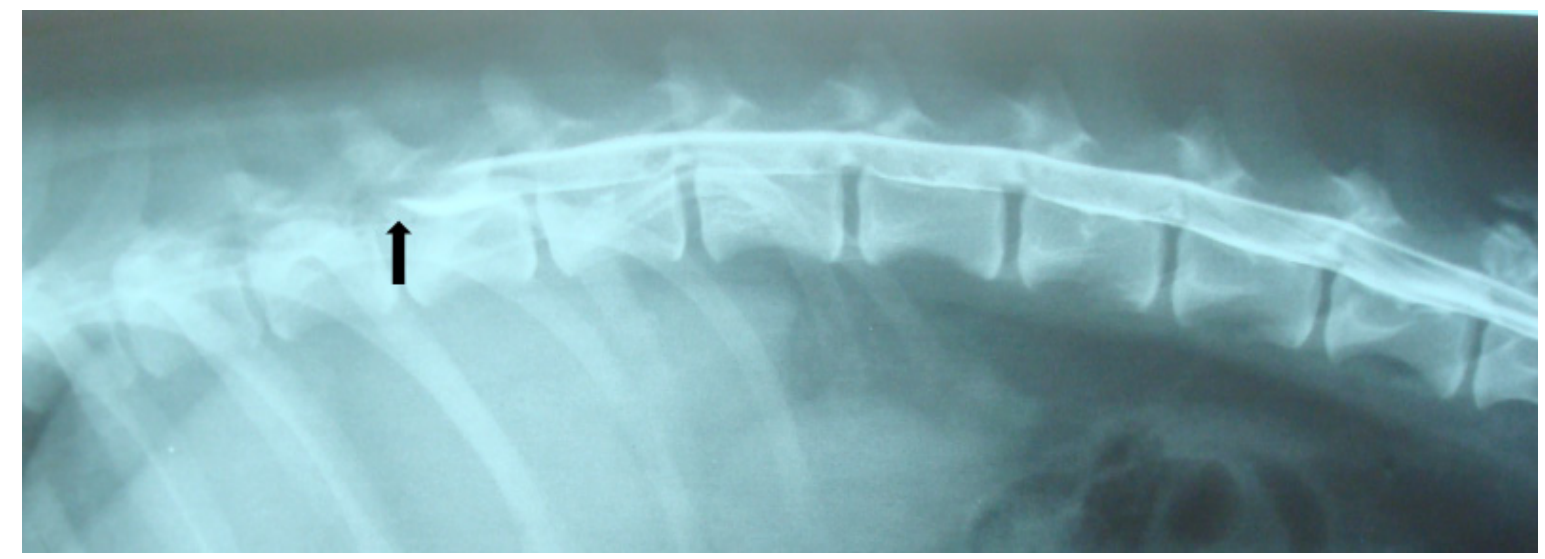

Fonte: Elaboração dos autores.

Figura 2. Imagem de mielografia lombar com elevação da coluna de contraste (seta preta) entre a primeira (L1) e segunda (L2) vértebras lombares e desaparecimento da coluna de contraste (seta vermelha) entre a décima segunda (T12) e décima terceira (T13) vértebras torácicas, que evidencia a lateralização da lesão em um cão com protrusão e extrusão do disco intervertebral, respectivamente.

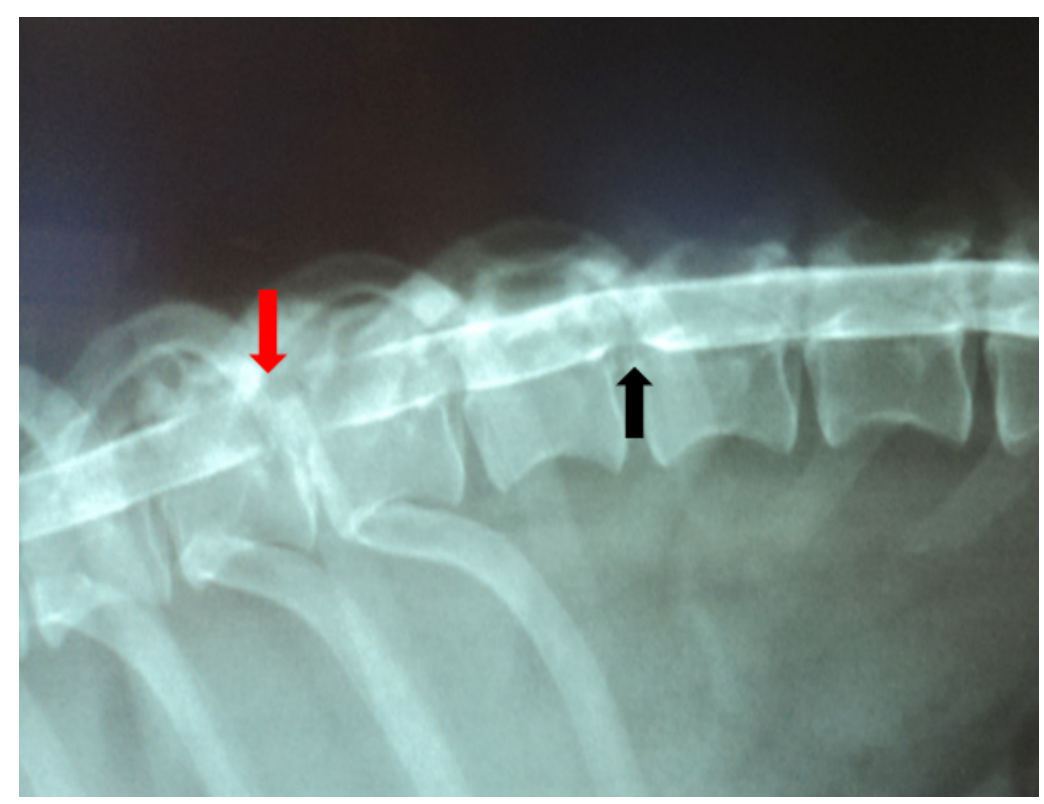

Fonte: Elaboração dos autores. 


\section{Resultados e Discussão}

A degeneração do disco intervertebral é um processo normal que se desenvolve com a idade do animal (BRISSON, 2010), mas que posteriormente pode ocasionar a doença do disco intervertebral (OLBY; JEFFERY, 2012). A doença do disco intervertebral toracolombar é a causa mais comum de disfunção neurológica em pequenos animais (DEWEY, 2013).

Os locais mais comumente envolvidos com extrusão de disco toracolombar são os espaços discais intervertebrais entre T11 e L2. Estes locais perfazem aproximadamente 65 a $75 \%$ de todas as extrusões discais. A extrusão do disco intervertebral toracolombar ocorre primariamente em raças condrodistróficas, como os Dachshunds, Pequines, Beagle, Poodle miniatura e toy, Shih-tzus e Lhasa apsos. Os Dachshunds têm 10 vezes mais riscos que todas as outras raças combinadas e cerca de $80 \%$ dos problemas ocorrem em animais entre 3 e 7 anos de idade (DEWEY, 2013). Tais afirmações corroboram com os resultados encontrados no presente estudo, a qual mostrou que $85 \%$ dos pacientes apresentavam compressão medular entre T11 e L2, sendo o espaço intervertebral entre a décima segunda e décima terceira vértebras torácicas (T12-T13) o mais acometido (40\%). Os animais da raça Dachshunds foram os mais afetados $(75 \%)$, com idade média entre 5 e 6 anos, seguidos dos animais sem raça definida (15\%), Shih-tzus (5\%) e Basset hound $(4 \%)$.

Em geral, a punção subaracnóide é facilmente colocada em prática na cisterna cerebelomedular e a injeção cisternal permite uma excelente visão da coluna vertebral cervical. A principal limitação da injeção na cisterna está no fato de que a distribuição do meio de contraste em sentido caudal pela espinha depende da gravidade e do fluxo de LCE. O agente de contraste pode não fluir além de uma lesão espinhal se o espaço subaracnóide estiver significativamente comprimido e ocluído. Como consequência, tem-se o fluxo de contraste direcionado para o encéfalo, aumentando o risco de convulsões (TOOMBS; WATERS, 2007). No estudo em questão observouse que apenas um dos 20 pacientes submetidos à mielografia lombar apresentou convulsão após o exame, o que representa $5 \%$ dos pacientes. Isso pode ser justificado pelo fato do paciente prossivelmente apresentar um limiar convulsivógeno baixo, pois apesar da dose intermediária utilizada, a crise foi inevitável. Esse resultados concordam com Barone et al. (2002), os quais relatam que 5\% dos pacientes do seu estudo apresentaram convulsão pós mielografia lombar.

Para minimizar os efeitos neurotóxicos sem prejudicar a efetividade do exame mielográfico, o agente de contraste ideal deve ser radiopaco em concentrações seguras, hidrossolúvel, miscível com o líquido cerebroespinhal (LCE), atóxico e removido do LCE por processos fisiológicos, mas não tão rapidamente a ponto de anular a obtenção das imagens radiográficas (DA COSTA; PARENT; DOBSON, 2011). Os meios mais utilizados na medicina veterinária são o iohexol e o ioversol (TUDURY et al., 1997). Ambos são excretados pelos rins até 48 horas após a injeção (KEALY; McALLISTER, 2005). No presente estudo, o iohexol foi utilizado em todos os pacientes e apresentou-se eficaz tanto na sua visibilização nas radiografias como em relação a sua neurotoxicidade.

Apesar da posição em decúbito esternal com flexão dos membros pélvicos permitir maior abertura entre as lâminas de L5 e L6 (PUGGIONI et al., 2006) que facilitaria a inserção da agulha no momento da punção, o decúbito lateral e semi-flexão dos membros pélvicos utilizados no referido estudo foi suficiente para que a punção fosse realizada com eficácia.

Foi observado epidurografia em três $(15 \%)$ pacientes, sendo que apenas um apresentou falha para a localização da lesão, e os três para lateralização da lesão. Segundo Barone et al. (2002), uma das principais complicações observadas com a punção lombar na realização de mielografias é a realização 
inadvertida da epidurografia. Segundo Toombs e Waters (2007), a vantagem da injeção lombar repousa na circulação anterógrada do meio de contraste sob pressão da injeção, podendo ser impulsionado em torno das lesões indutoras de obstrução ao fluxo do LCR. A desvantagem da injeção através do espaço subaracnóide lombar está na dificuldade de execução e, consequentemente, na possibilidade de injeção inadvertida ou extravasamento do meio de contraste para o espaço epidural, provocando artefatos e imagens inconclusivas.
A localização da lesão foi possível em 95\% dos pacientes submetidos ao exame de mielografia lombar (Tabela 1). Esses dados são superiores aos resultados de Bos et al. (2012), que relataram acurácia de 90,9\% para a localização do local. Bos et al. (2012) em contrapartida aos resultados encontrados com a mielografia, relataram $100 \%$ de acurácia para a localização e lateralização da lesão utilizando ressonância magnética.

Tabela 1. Parâmetros avaliados e o número de cães (porcentagem) com extrusão do disco intervertebral toracolombar submetidos ao exame de mielografia por punção lombar.

\begin{tabular}{lll}
\hline \multicolumn{1}{c}{ Parâmetro Avaliado } & Presença & $\begin{array}{c}\text { Número de Animais (\%) } \\
\text { Ausência }\end{array}$ \\
\hline Eficácia na localização da lesão & $19(95)$ & $1(5)$ \\
Eficácia na lateralização da lesão & $12(60)$ & $8(40)$ \\
Complicações & & \\
Convulsão & $1(5)$ & $19(95)$ \\
Deterioração neurológica & $0(0)$ & $20(100)$ \\
Epidurografia & $3(15)$ & $17(85)$ \\
Canalograma & $0(0)$ & $0(0)$ \\
Derrame Subdural & $0(0)$ & $0(0)$ \\
\hline
\end{tabular}

Fonte: Elaboração dos autores.

Dentre os 20 pacientes analisados, três apresentavam compressão medular em mais de um ponto caracterizados por lesões entre T11-T12 e T12-T13; T12-T13 e T13-L1; e T11-T12 e L1-L2. Segundo Brisson (2010), a metaplasia condróide pode ocorrer ao longo dos discos de toda a coluna vertebral. Um total de 24 a $90 \%$ dos cães da raça Dachshund desenvolvem mineralização de um ou mais discos intervertebrais, com uma média de 2,3 discos calcificados por cão. Calcificações foram relatadas em todos os discos, mas os discos da região torácica caudal, especialmente entre T10 e T13, costumam ser mais afetados (BRISSON, 2010).

Oito padrões ventrodorsais de contraste mielográfico foram relatados em cães de raças pequenas com extrusão do disco intervertebral confirmada. Seis dos oito padrões foram consistentes com a lateralização ou extrusão ventrolateral, enquanto que dois foram consistentes com localização ventral da extrusão. A precisão relatada na projeção ventrodorsal neste estudo foi de $89 \%$. Em $83 \%$ dos cães com lacunas de contraste desiguais, o material do disco foi encontrado no lado com a menor lacuna. Este fenômeno é denominado obstrução paradoxal do contraste mielográfico (BOS et al., 2004). Projeções oblíquas apresentam maior eficácia do que a projeção ventrodorsal para a localização circunferencial e é recomendada para todos os casos (KIRBERGER; ROOS; LUBBE, 1992; TANAKA; NAKAIAMA; TAKASE, 2004; GIBBONS et al., 2006). O uso de projeções oblíquas e ventrodorsais são consideradas mais 
úteis do que apenas uma delas para a lateralização da lesão (BRISSON, 2010). No presente estudo, foram utilizadas projeções ventrodorsais e oblíquas, porém, em $40 \%$ dos pacientes não foi possível identificar a lateralização da lesão. Esses dados concordam com os resultados encontrados por Bos et al. (2012), os quais observaram a eficácia da mielografia na lateralização da lesão em 54,6\% dos pacientes.

Dos pacientes que apresentaram lateralização da lesão confirmada, 58,3\% apresentaram compressão do lado esquerdo e 41,6 \% do lado direito (Tabela $1)$.

É comum que os cães com extrusão aguda do disco intervertebral apresentem imagem da medula espinhal no exame mielográfico com aspecto de inchaço, este processo é ocasionado por um edema medular. Este edema pode obliterar o espaço subaracnóide e, por consequência, impedir ou reduzir a passagem do meio de contraste no âmbito da lesão e às vezes até mesmo em uma área maior. A injeção lombar de contraste gera uma pressão que ajuda a neutralizar o efeito do edema medular, permitindo que o meio de contraste possa fluir através das partes estreitas do espaço subaracnóide de modo que o local do edema possa ser mais claramente demostrado (TAEYMANS; SAUNDERS; BREE, 2002).

De acordo com Kirberger; Roos e Lubbe (1992), a injeção lombar do contraste em menos de dez segundo e a obtenção de radiografias imediatamente a pós a aplicação delimita melhor a área de edema da medula espinhal. No nosso estudo, a injeção do contraste foi realizada de forma lenta sem interferir na delimitação do edema, evitando então a aplicação inadvertida do mesmo no espaço subaracnóide. Para Taeymans, Saunders e Bree (2002), a aplicação rápida do contraste sob pressão pode deslocar a agulha do espaço subaracnóide e ocorrer formação de epidurografia.

As primeiras radiografias foram realizadas imediatamente após injeção do contraste em todos os pacientes do estudo. Segundo Taeymans, Saunders e Bree (2002), o aquecimento do meio de contraste para minimizar a sua viscosidade, inclinando ou rolando o animal para promover o fluxo médio do contraste, e o encurtamento do tempo médio entre a injeção lombar do contraste e a primeira exposição radiográfica podem influenciar na quantidade de edema da medula espinal visível na mielografia.

Com base no estudo, conclui-se que a mielografia lombar foi muito útil para o diagnóstico das extrusões dos discos intervertebrais toracolombares tendo acurácia de $95 \%$ e $60 \%$ para a localização e a lateralização da lesão respectivamente, com complicações mínimas.

\section{Aspectos Éticos e de Biossegurança}

Todos os procedimentos foram aprovados pela Comissão de Ética no Uso de Animais (CEUA) da FCAV - Unesp - Jaboticabal, sob o protocolo $n^{\circ}$ 007892/12.

\section{Referências}

ALLAN, G. S.; WOOD, A. K. W. Iohexol myelography in the dog. Veterinary Radiology \& Ultrasound, Virginia, v. 29 , n. 2, p. $78-82,1988$.

BARONE, G.; ZIEMER, L. S.; SHOFER, F. S.; STEINBERG, S. A. J. Risk factors associated with development of seizures after use of iohexol for myelography in dogs: 182 cases (1998). Journal of the American Veterinary Medical Association, Chicago, v. 220, n. 10, p. 1499-502, 2002.

BESALTI, O.; PEKCAN, Z.; SIRIN, Y. S.; ERBAS, G. Magnetic resonance imaging findings in dogs with thoracolumbar intervertebral disk disease: 69 cases (1997-2005). Journal of the American Veterinary Medical Association, Chicago, v. 228, n. 6, p .902-908, 2006.

BOS, A. S.; BRISSON, B. A.; HOLMBERG, D. L.; NYKAMP, S. G. Use of the ventrodorsal myelographic view to predict lateralization of extruded disk material in small-breed dogs with thoracolumbar intervertebral disk extrusion: 104 cases (2004-2005). Journal of the American Veterinary Medical Association, Chicago, v. 230, n. 12, p. 1860-1865, 2007. 
BOS, A. S.; BRISSON, B. A.; NYKAMP, S. G.; POMA, R.; FOSTER, R. A. Accuracy, intermethod agreement, and inter-reviewer agreement for use of magnetic resonance imaging and myelography in small-breed dogs with naturally occurring first-time intervertebral disk extrusion. Journal of the American Veterinary Medical Association, Chicago, v. 240, n. 8, p. 969-977, 2012.

BRISSON, B.A. Intervertebral disc disease in dog. Veterinary Clinics of North America. Small Animal Practice, Philadelphia, v. 40, n. 5, p. 829-858, 2010.

DA COSTA, R. C.; DOBSON, H.; PARENT, J. $M$. Incidence of and risk factors for seizures after myelography performed with iohexol in dogs: 503 cases (2002-2004). Journal of the American Veterinary Medical Association, Chicago, v. 238, n. 10, p. 1296-1300, 2011.

DEWEY, C. W. Surgery of the thoracolumbar spine. In: FOSSUM, T. W. Small animal surgery. 4. ed. Missouri: Elseviar, 2013. cap. 41, p. 1508-1528.

GIBBONS, S. E.; MACIAS, C.; DE STEFANI, A.; PINCHBECK, G. L.; McKEE, W. M. The value of oblique versus ventrodorsal myelographic views for lesion lateralization in canine thoracolumbar disc disease. Journal of Small Animal Practice, Ontario, v. 47, n. 11, p. 658-662, 2006.

ISRAEL, S. K.; LEVINE, J. M.; KERWIN, S. C.; LEVINE, G. J.; FOSGATE, G. T. The relative sensitivity of computed tomography and myelography for identification of thoracolumbar intervertebral disk herniations in dogs. Veterinary Radiology \& Ultrasound, Virginia, v. 50, n. 3, p. 247-252, 2009.

JESEN, V. F. Asymptomatic radiographic disappearance of calcified intervertebral disc material in the dachshund. Veterinary Radiology and Ultrasound, Virginia, v. 42, n. 2, p. 141-748, 2001.

KEALY, J. K.; McALLISTER, H. O Crânio e a coluna vertebral. In:__. Radiologia e ultra-sonografia do cão e do gato. 3. ed. Barueri: Manole, 2005. p. 339-384.

KIRBERGER, R. M.; ROOS, C. J.; LUBBE, A. M. The radiological diagnosis of thoracolumbar disc disease in the dachshund. Veterinary Radiology \& Ultrasound, Virginia, v. 33, n. 5, p. 255-261, 1992.

KISHIMOTO, M.; YAMADA, K.; UENO, H.; KOBAYASHI, Y.; WISNER, E. R. Spinal cord effects from lumbar myelographic injection technique in the dog. The Journal Veterinary Medical Science, Tokio, v. 66, n. 1, p. 67-69, 2004.

LEVINE, J. M.; LEVINE, G. J.; BOOZER, L.; SCHATZBERG, S. J.; PLATT, S. R.; KENT, M.; KERWIN, S. C.; FOSGATE, G. T. Adverse effects and outcome associated with dexamethasone administration in dogs with acute thoracolumbar intervertebral disk herniation: 161 cases (2000-2006). Jounal of American Veterinary Medical Association, Chicago, v. 232, n. 2, p. 411-417, 2008.

LEWIS, D. D.; HOSGOOD, G. Complications associated with the use of iohexol for myelography of the cervical vertebral column in dogs: 66 cases (1988-1990). Journal of the American Veterinary Medical Association, Chicago, v. 200, n. 9, p. 1381-1384, 1992.

MCCARTNEY, W. T. Lumbar myelography in 79 dogs, using different puncture sites. Veterinary Record, Londres, v. 141, n. 16, p. 417-419, 1997.

NAUDE, S. H.; LAMBRECHTS, N. E.; WAGNER, W. M.; THOMPSON, P. N. Association of preoperative magnetic resonance imaging findings with surgical features in dachshunds with thoracolumbar intervertebral disk extrusion. Journal of the American Veterinary Medical Association, Chicago, v. 232, n. 5, p. 702-708, 2008.

NEWCOMB, B.; ARBLE, J.; ROCHA, T. M.; PECHMAN, R.; PAYTON, M. Comparison of computed tomography and myelography to a reference standard of computed tomographic myelography for evaluation of dogs with intervertebral disc disease. Veterinary Surgery, Davis, v. 41, n. 2, p. 207-214, 2012.

OLBY, N.; HOULTON, J. E. Correlation of plain radiographic and lumbar myelographic findings with surgical findings in thoracolumbar disc disease. Journal of Small Animal Practice, Ontario, v. 35, n. 7, p. 345-350, 1994.

OLBY, N.; JEFFERY, N. Pathogenesis and physiology of central nervous system disease and injury. In: TOBIAS, K. M.; JOHNSTON, S. A. Veterinary surgery. Small animal. Missouri: Elsevier Saunders, 2012. v. 1, p. 374387.

PUGGIONI, A.; ARNETT, R.; CLEGG, T.; GLYDE, M.; TOBIN, E.; McALLISTER, H. Influence of patient positioning on the L5-L6 mid-laminar distance. Veterinary Radiology \& Ultrasound, Virginia, v. 47, n. 5, p. 449-452, 2006.

ROBERTS, R. E.; SELCER, B. A. Myelography and epidurography. Veterinary Clinics of North America: Small Animal Practice, Philadelphia, v. 23, n. 2, p. 307329, 1993.

SCHULZ, K. S.; WALKER, M.; MOON, M.; WALDRON, D.; SLATER, M.; McDONALD, C. E. Correlation of clinical, radiographic, and surgical localization of intervertebral disc extrusion in smallbreed dogs: a prospective study of 50 cases. Veterinary Surgery, Davis, v. 27, n. 2, p. 105-11, 1998. 
SHARP, N. J. H.; WHEELER, S. Diagnostic aids. In: Small animal spinal disorders. 2. ed. Edinburgh: Elsevier Mosby, 2005. p. 42-72.

TAEYMANS, O.; SAUNDERS, J. H.; BREE, H. N. Radiology corner canine myelography. Veterinary Radiology \& Ultrasound, Virginia, v. 43, n. 6, p. 550$551,2002$.

TANAKA, H.; NAKAYAMA, M.; TAKASE, K. Usefulness of myelography with multiple views in diagnosis of circumferential location of disc material in dogs with thoracolumbar intervertebral disc herniation. The Journal of Veterinary Medical Science, v. 66, n. 7, p. 827-833, 2004.
TOOMBS, J. P.; WATERS, D. J. Afecção do disco intervertebral. In: SLATTER, D. Manual de cirurgia de pequenos animais. 3. ed. São Paulo: Manole, 2007. v. 1, p. 1193-1208.

TUDURY, E. A.; BAHR ARIAS, M. V.; CAMARGO, P. L.; FARIA, M. L. E.; MACHADO, C. E. G. Meio de contraste ioversol em neuroradiologia canina. Ciência Rural, Santa Maria, v. 27, n. 4, p. 619-623, 1997.

WHEELER, S. J.; DAVIES, J. V. Iohexol myelography in the dog and cat: a series of one hundred cases, and a comparison with metrizamide and iopamidol. Journal of Small Animal Practice, Ontario, v. 26, n. 5, p. 247-256, 1985. 
